\title{
Incisional atrial reentrant tachycardia: Experimental study on the conduction property through the isthmus
}

Yosuke Ishii, $M D^{\mathrm{a}}$

Takashi Nitta, MD

Shun-ichiro Sakamoto, MD

Shigeo Tanaka, MD ${ }^{\mathrm{a}}$

Goro Asano, $\mathrm{MD}^{\mathrm{b}}$
From the Departments of Cardiothoracic Surgery ${ }^{\mathrm{a}}$ and Pathology, ${ }^{\mathrm{b}}$ Nippon Medical School, Tokyo, Japan.

Received for publication May 29, 2002; revisions requested Aug 15, 2002; revisions received Sept 4, 2002; accepted for publication Sept 9, 2002

Address for reprints: Yosuke Ishii, MD, 1-1-5 Sendagi, Bunkyo-ku, Tokyo 113-8603, Japan (E-mail: yosuke-i@jb3.so-net.ne.jp).

J Thorac Cardiovasc Surg 2003;126:254-62

Copyright $(\odot) 2003$ by The American Association for Thoracic Surgery

$0022-5223 / 2003 \$ 30.00+0$

doi:10.1016/S0022-5223(02)73603-9
Background: Incisional atrial reentrant tachycardia is a life-threatening tachyarrhythmia after surgery for congenital heart disease. Slow conduction through an isthmus between anatomical barriers, such as a right atriotomy or the sites for cannulation, has been shown to be a prerequisite for perpetuation of the incisional atrial reentrant tachycardia. However, the conduction property through the isthmus has not been examined in detail.

Methods: To examine the conduction property, 2 tandem incisions were made on the lateral right atrium with various distances (3 to $20 \mathrm{~mm}$ ) between the incisions in 16 canines. Four weeks after the surgery, the lateral right atrium was mapped epicardially during pacing to examine the conduction property through the isthmus. The conduction property was characterized by approximated curves of the conduction velocity through the isthmus in accordance with the pacing cycle lengths. The atrial tissue at the isthmus was examined microscopically.

Results: The approximated curves of the conduction velocity were classified into 3 different types. Decremental conduction was observed only in the isthmi between 5 and $15 \mathrm{~mm}$ in width. A small amount of surviving myocardium between the scars formed the critical isthmus microscopically (decremental type). In the isthmi wider than $15 \mathrm{~mm}$ in width, slow conduction was not seen at any paced cycle length (nondecremental type). In the extremely narrow isthmi less than $5 \mathrm{~mm}$ in width, all of the atrial myocardium at the isthmus was replaced by fibrous tissue. Conduction was blocked at the isthmus and the activation detoured around the incisions (block type). There was a statistically significant difference in the approximated curves between the 3 different types of conduction properties $(P<.01)$.

Conclusion: The width of the isthmus determines the conduction property through the isthmus that contributes to the development of the incisional atrial reentrant tachycardia. Thus, the incisional atrial reentrant tachycardia may be preventable by leaving a sufficient amount of surviving myocardium between the incisions or by connecting the incisions by an ablative procedure.

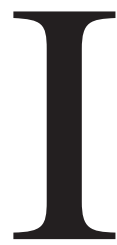

ncisional atrial reentrant tachycardia (IART) is a life-threatening complication after surgery for congenital heart disease. The incidence of the IART has been described to be about $20 \%$ patients with a 10-year follow-up period after the Fontan procedure. ${ }^{1,2}$ Also in patients who have undergone atrial septal defect repair during childhood, it has been reported that the tachycardia occurs in $4 \%$ to $18 \%$ of these patients during a 10 - to 32 -year follow-up period after the procedure. ${ }^{3,4}$ These patients suffer from palpitations, fatigue, dyspnea, syncope, or hemodynamic collapse during the tachycardia. It even results in sudden cardiac death in patients with a rapid ventricular response or with 
impaired ventricular function. ${ }^{5,6}$ Pharmacological treatment frequently fails to control this tachyarrhythmia. ${ }^{7}$ Therefore, surgical intervention or prevention of the IART has important implications.

There are several different reentrant circuits in IART. Lesh and colleagues described the reentrant circuit of IART, which included an isthmus between 2 anatomical barriers, such as atriotomy or cannulation site. ${ }^{8,9}$ The right atrial scars or atrial septal baffle after the Fontan procedure can also be a substrate responsible for IART. ${ }^{10-12}$ Most of these reentrant circuits have been shown to have slow conduction in the critical isthmus between the surgical scars or anatomical barriers. Presence of slow conduction in the reentrant circuit allows sufficient time for the myocardium to recover, allowing a stable reentrant activation for IART to occur. ${ }^{8}$

IART does not necessarily occur in all patients after surgery for congenital heart disease. In addition to the anatomical substrates for IART, an atrial scar caused by an atriotomy or elevated atrial wall pressure could provide a condition prone to initiate the IART. ${ }^{8-12}$ However, the conduction property through the isthmus has not been examined in detail. Precautions against the IART during initial surgery have not been raised either.

We hypothesized that the width of the isthmus was involved in the determination of the conduction property through the isthmus. We focused on IART, in which the reentrant circuit was composed of the isthmus between the incisions on the lateral right atrium. The purpose of this study was to examine whether the width of the isthmus determined the conduction property.

\section{Materials and Methods \\ Surgical Preparation}

All animals received humane care in compliance with the "Principles of Laboratory Animal Care," formulated by the National Society for Medical Research, and the "Guide for the Care and Use of Laboratory Animals," prepared by the National Academy of Science and published by the National Institute of Health (NIH Publication 86-23, received 1985). In addition, this study protocol was approved by the Animal Ethics Committee of Nippon Medical School. Adult mongrel canines weighting 16 to $34 \mathrm{~kg}(\mathrm{n}=16)$ were anesthetized with intravenous thiamylal sodium $(10 \mathrm{mg} / \mathrm{kg})$, intubated with a cuffed endotracheal tube, and mechanically ventilated with a volume-controlled respirator. An adequate level of anesthesia was maintained by an intermittent infusion of $5 \mathrm{mg}$ midazolam. A limb-lead electrocardiogram was monitored. After a right lateral thoracotomy through the fourth intercostal space was made, the pericardium was opened and the lateral right atrium was exposed. Two tandem incisions were made on the lateral right atrium (Figure 1). The length of each incision was adjusted to be $30 \mathrm{~mm}$. An isthmus between the incisions was created with the width varying in a range from 3 to $20 \mathrm{~mm}(3,5,7,10,12,15,18$, or $20 \mathrm{~mm}$ ) in each canine. These incisions were made by the closed heart technique without the use of cardiopulmonary bypass as described previously. ${ }^{13}$ After the right lung was reexpanded, the

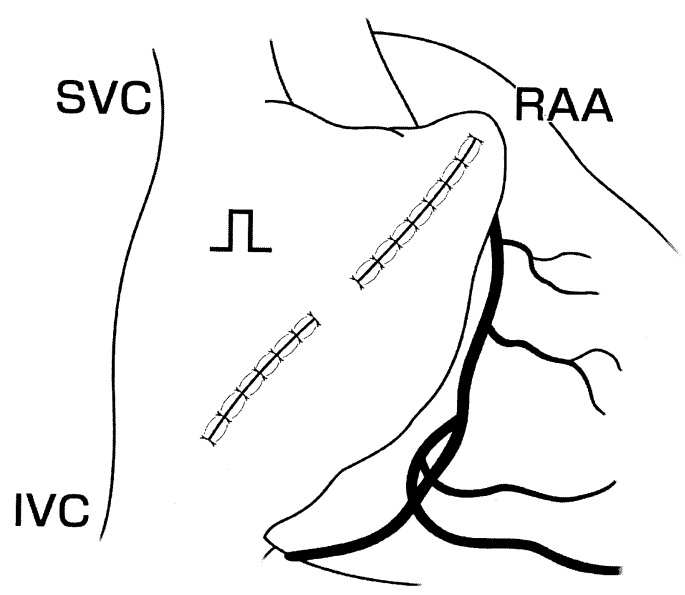

Figure 1. Two tandem incisions made on the lateral right atrium without the use of cardiopulmonary bypass. The length of each incision was adjusted to $30 \mathrm{~mm}$. An isthmus between the incisions was created with the width adjusted to range from $3 \mathrm{~mm}$ to $20 \mathrm{~mm}$ in each canine. The rectangle indicates the pacing site. $R A A$, Right atrial appendage; $S V C$, superior vena cava; IVC, inferior vena cava.

chest was closed in layers. The canines were injected with an intramuscular antibiotic (ceftazidime $0.25 \mathrm{~g} / \mathrm{d}$ ) injection for 3 days postoperatively. Four weeks after the initial surgery, the canines were anesthetized again with intravenous pentobarbital sodium (30 $\mathrm{mg} / \mathrm{kg}$ ), intubated with a cuffed endotracheal tube, and mechanically ventilated with a volume-controlled respirator. An adequate level of anesthesia was maintained by an intermittent infusion of 1 to $2 \mathrm{mg}$ pentobarbital sodium. A limb-lead electrocardiogram was monitored. The arterial blood gas, electrolytes, and PH were measured at least twice during the experiment. Those measurement values were corrected within the limits of normal. After a median sternotomy was made, the pericardium was opened and the lateral right atrium was exposed. The conduction property of the isthmus was evaluated in 2 ways: by the activation map of the lateral right atrium and the conduction velocity through the isthmus. After completion of all the electrophysiological measurements, the tissue in the isthmus was examined microscopically to determine the width and amount of the surviving myocardium.

\section{Pacing Protocol}

A custom-made bipolar pacing electrode was placed on the lateral side of the isthmus in a fashion that the stimulation wave front passed through the isthmus at right angles to the 2 tandem incisions. The intraelectrode distance of the bipolar pacing electrodes was $1 \mathrm{~mm}$. Stimulation was performed using a programmable pulse generator (Cardiac Stimulator, Fukuda Denshi Corp, Tokyo, Japan). Continuous pacing was conducted at a cycle length from $350 \mathrm{~ms}$ to the local refractory period in 10-ms decrements. The stimulus output was set at twice the pacing threshold.

\section{Activation Mapping of the Lateral Right Atrium}

The lateral right atrium was mapped during continuous pacing with a custom-made electrode patch that carried 253 unipolar 


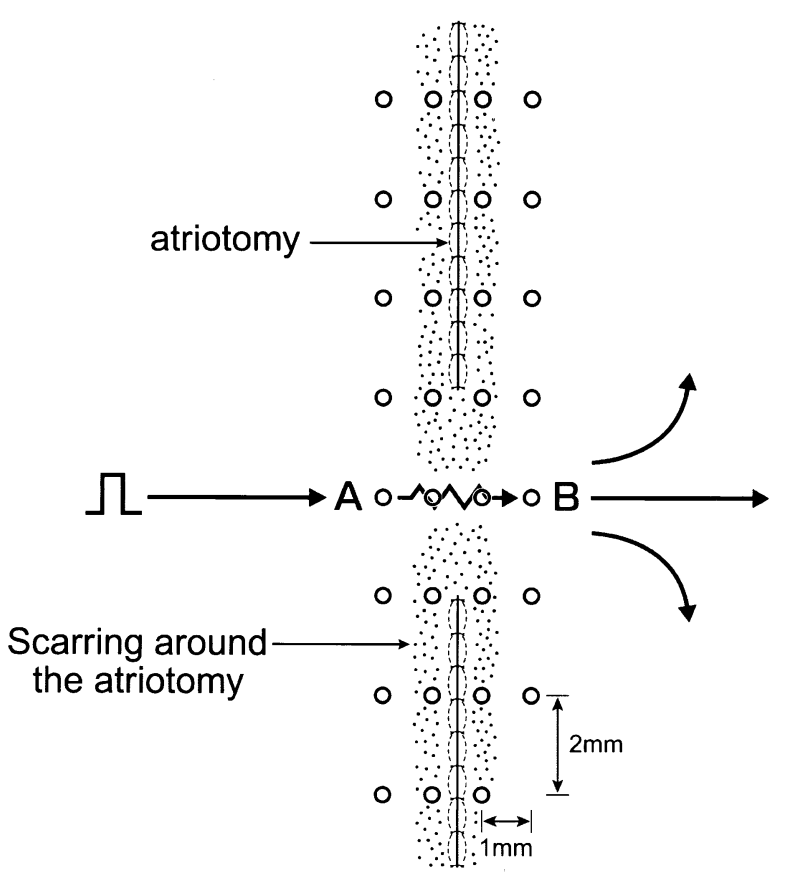

Figure 2. Calculation of the conduction velocity through the isthmus. Conduction velocity $(\mathrm{m} / \mathrm{s})=$ Distance (electrode $A$ - electrode B: $3 \mathrm{~mm}$ )/Activation time difference (ms). The white circles indicate the 31 bipolar electrodes. The distance between point $A$ and point $B$ is $3 \mathrm{~mm}$. The rectangle indicates the pacing site. The arrows represent the activation wave front through the isthmus. The small black points indicate scarring around the incisions.

electrodes. The electrode patch was constructed from a form-fitting silicon elastomer (Specialty Silicone Fabricators Inc, Paso Robles, Calif) and silver electrodes $0.5 \mathrm{~mm}$ in diameter (Pacific Wire \& Cable, Santa Ana, Calif). The interelectrode distance between the unipolar points was $1 \mathrm{~mm}$. The electrode patch was placed on the lateral right atrium. Limb-lead electrocardiograms were simultaneously recorded. Atrial activation sequence data were recorded during a spontaneous rhythm and continuous pacing. The data were acquired with a 256-channel computerized data-acquisition and data-analysis system based on a high-performance graphics workstation (Indigo 2, Silicon Graphics Inc, Mountain View, Calif). The system was run with custom-made software that was capable of data acquisition control, management, display, and analysis. Unipolar electrograms were recorded at a gain of 500 with a frequency response of 0.5 to $500 \mathrm{~Hz}$. Each channel was digitized at $2000 \mathrm{~Hz}$ with a 12-bit resolution. Local activation times were determined as the time of the maximum negative derivative $(-\mathrm{dV} / \mathrm{dt})$ of the unipolar electrograms. All electrograms were edited visually to verify accuracy of the computer-selected activation times. Computer-generated activation sequence maps were constructed from all recordings. Data processing and a 3-dimensional interactive display were performed on the graphic workstation. Activation maps were constructed on a 3-dimensional surface model of the canine atrium displayed on the computer. A site of conduction block was defined as the site between any 2 adjacent electrodes having an activation time difference of $>10$ $\mathrm{ms} / \mathrm{mm}$.

\section{Conduction Velocity Through the Isthmus}

The conduction velocity through the isthmus was measured by using a custom-made electrode patch that carried 31 bipolar electrodes, which was constructed from a form-fitting silicon elastomer and silver electrodes as described above. The transverse and longitudinal interelectrode distances between the bipolar electrodes were $1 \mathrm{~mm}$ and $2 \mathrm{~mm}$, respectively. The intraelectrode distance between each bipolar electrode was $1 \mathrm{~mm}$. Limb-lead electrocardiograms were simultaneously recorded. Data were recorded during continuous pacing as described above. Bipolar electrograms were recorded at a gain of 500 with a frequency response of 5 to $500 \mathrm{~Hz}$. The local activation time was defined as the time of the maximum absolute amplitude of the bipolar electrograms. Activation maps were constructed on the graphic workstation to confirm the location of the electrodes and isthmus. The electrode patch was placed on the lateral right atrium in a fashion so that the center of the electrode array was placed just at the center of the isthmus between the 2 incisions. The conduction velocity was calculated as the distance $(3 \mathrm{~mm})$ divided by the activation time difference between the 2 electrodes that were selected from both lateral sides of the 4 electrode lines (Figure 2). The location of the isthmus was confirmed by the bipolar activation map, and then the 2 electrodes with the shortest activation time difference were identified. If the conduction block at the isthmus was identified by the unipolar activation map, the activation time difference was measured between the electrode with the earliest activation time and the axisymmetric electrode of the incisions. Thus, the calculated conduction velocity in the block pattern was not through the isthmus, but through a detoured pathway around the incisions. The conduction velocity was determined for each paced cycle length that was decreased from $350 \mathrm{~ms}$ to the local refractory period in $10 \mathrm{~ms}$ decrements. Then the conduction properties through the isthmus were characterized by plotting the conduction velocities in accordance with the pacing cycle lengths.

\section{Pathological Examination}

After completion of all the electrophysiologic measurements, the animals were euthanized and the lateral right atrium was excised for microscopic examination. The tissue blocks containing the isthmus were resected parallel to the tandem incisions. The tissues were fixed with $10 \%$ formalin solution and embedded in paraffin. The 2 markers were ticked off the tissue before fixing with formalin to examine the shrinkage of the tissue caused by formalin. The distance between the makers did not change before or after the fixation by formalin. Deparaffinized tissue sections were stained with hematoxylin-eosin and Elastica-Masson-Goldner. The amount of surviving atrial myocardium at the isthmus was measured microscopically. The measurement was processed digitally as follows. The tissue sections were observed under $4 \times$ magnification with light microscopy (AX 80, Olympus, Tokyo, Japan) and then directly captured by using a digital camera (DP 50, Olympus, Tokyo, Japan). Then, captured sections were converted to image files. Using the Photoshop software version 6.0 (Adobe Systems, San Jose, Calif), only the area of surviving myocardium at the isthmus was cut out. After binarization with an image processor, 

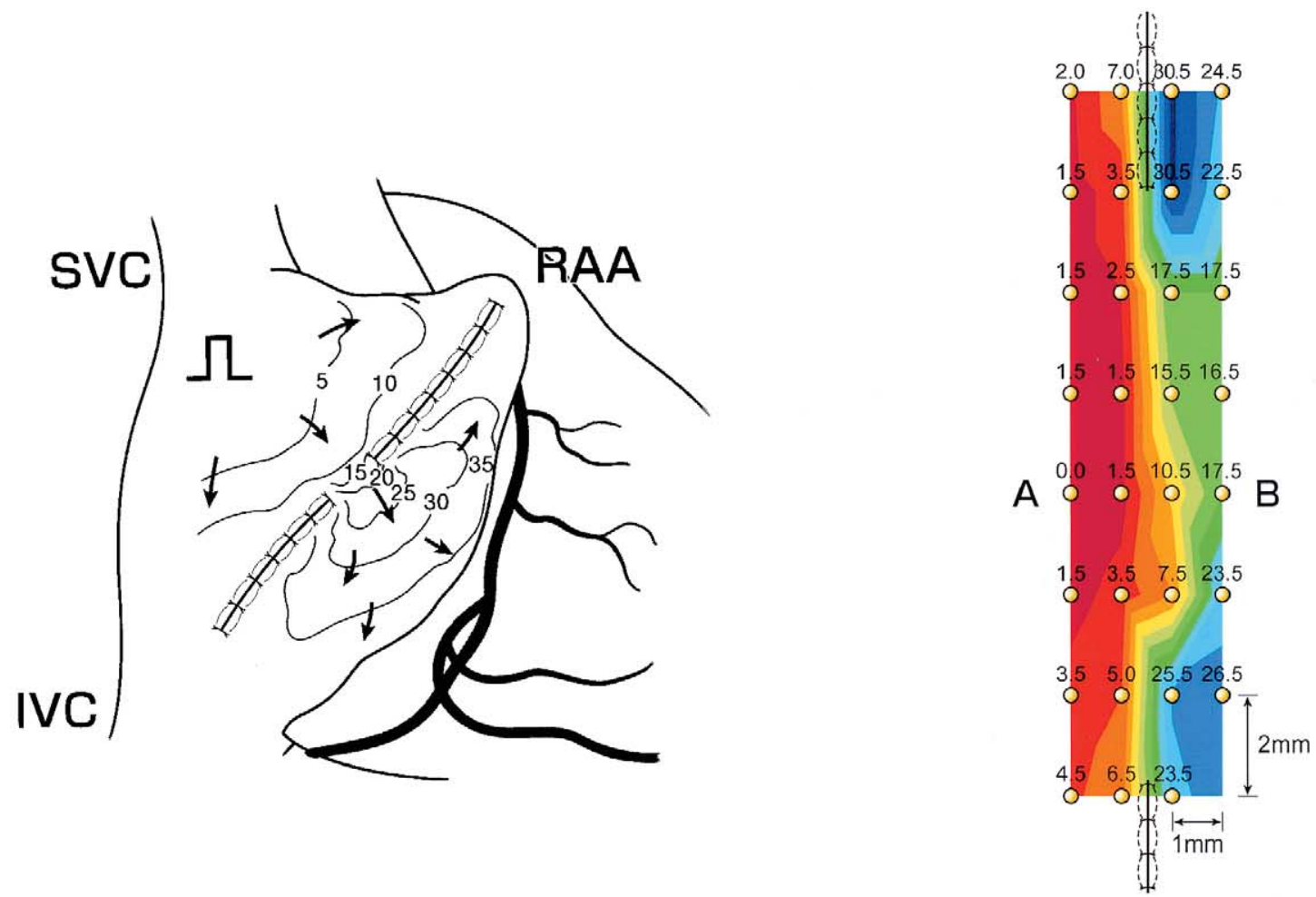

A

$\begin{array}{lllllll}\text { B } & 0 & 5 & 10 & 15 & 20 & 25\end{array}$

Figure 3. Activation map of the lateral right atrium and measurement of the conduction velocity through an isthmus of $12 \mathrm{~mm}$ in width. A, The activation map recorded by 253 unipolar electrodes during pacing at a cycle length of $200 \mathrm{~ms}$. Two tandem incisions are illustrated and the rectangle indicates the site of pacing. The arrows represent the activation sequence in the lateral right atrium. The crowded isochrones between the incisions suggest the presence of slow conduction at the isthmus. B, The activation map recorded by $\mathbf{3 1}$ bipolar electrodes during pacing at a pacing cycle length of $\mathbf{2 0 0} \mathbf{m s}$. The yellow points indicate the location of each electrode. The map is represented as color codes with $2.5 \mathrm{~ms}$ increments. In this example, the activation time difference between electrode $A$ and electrode $B$ is $17.5 \mathrm{~ms}$, thus the conduction velocity through the isthmus measured $0.17 \mathrm{~m} / \mathrm{s}$. Note that the activation extremely slowed when it penetrated the isthmus between the 2 adjacent electrodes (activation times $1.5 \mathrm{~ms}$ and $10.5 \mathrm{~ms}$ ). SVC, Superior vena cava; IVC, inferior vena cava; PV, pulmonary vein.

the area of surviving myocardium at the isthmus was measured by using the NIH Image version 1.61 analysis software. The correlation between the width of the isthmus determined preoperatively and the area of the surviving myocardium at the isthmus 4 weeks after the initial surgery was examined.

\section{Statistical Analysis}

All continuous values were expressed as mean \pm 1 standard deviation (SD). The approximated curves of the plots were compared between the various distances of the isthmus by using analysis of covariance (ANCOVA). We conducted ANCOVA, with a group factor of the conduction properties, as a covariate, and then a post hoc test was performed using Bonferoni's test. Linear regression analysis of the data was performed by the least squares method (model: surviving myocardium area $=a+b \times$ width of the isthmus).

\section{Results}

\section{Electrophysiological Findings}

Activation pattern of the lateral right atrium. Several different activation patterns were observed on the lateral right atrium during pacing. The activation patterns varied with the width of the isthmus and the paced cycle length. In the wider isthmi, more than $15 \mathrm{~mm}$ in width, the activation wave front passed through the isthmus without slow conduction during pacing at any cycle length. In the moderately narrow isthmi, between 5 and $15 \mathrm{~mm}$ in width, slow conduction through the isthmi was observed as shortening of the pacing cycle length (Figure 3,A). At a longer paced cycle length, the activation passed through the isthmus without slow conduction. At a shorter paced cycle length, 

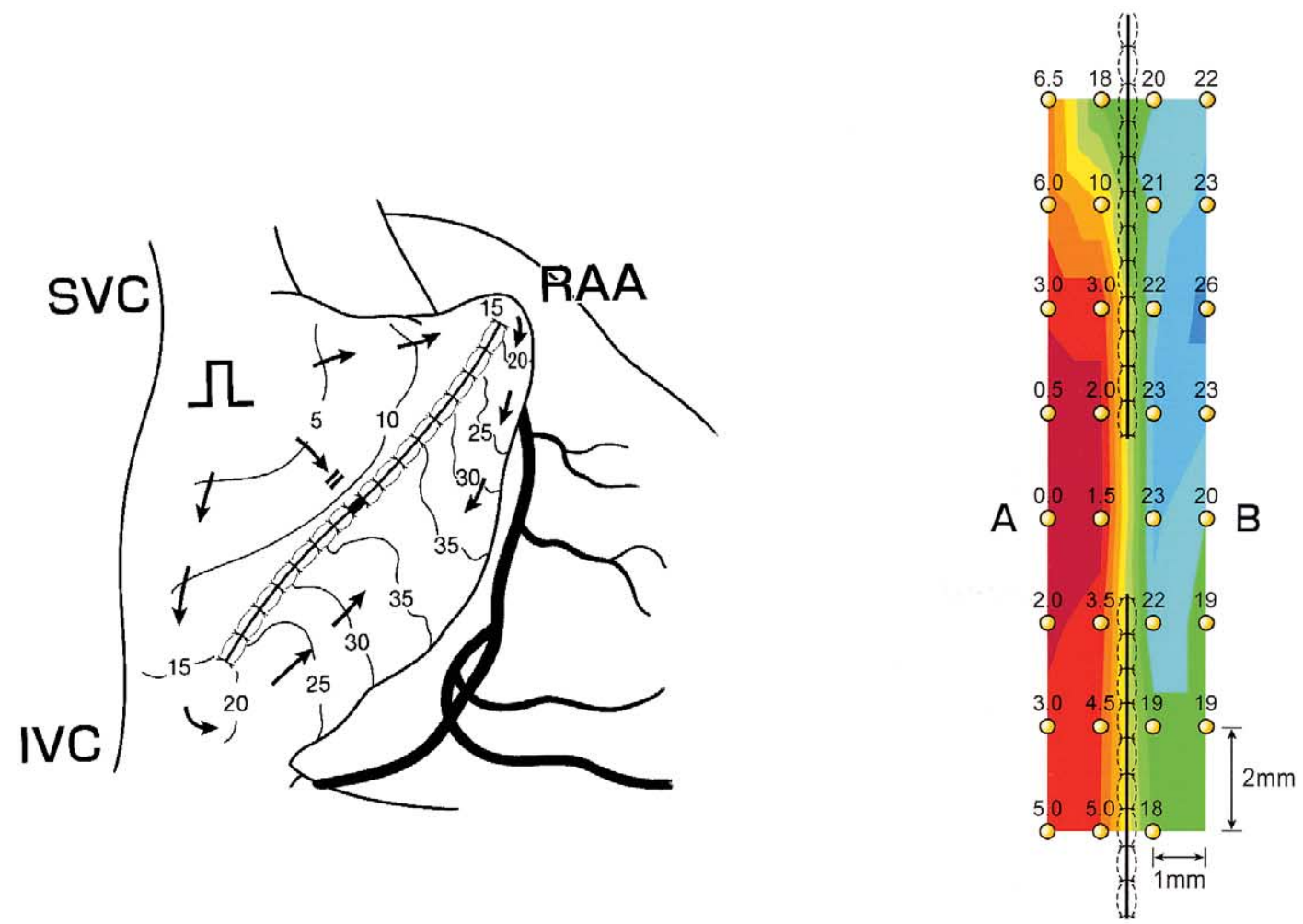

A

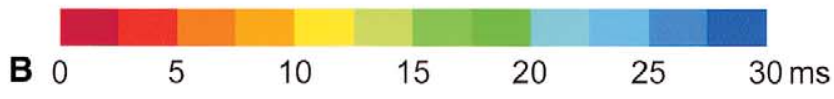

Figure 4. Activation map of the lateral right atrium and measurement of the conduction velocity through an isthmus of $3 \mathrm{~mm}$ in width. A, The activation map recorded by 253 unipolar electrodes during pacing at a pacing cycle length of $340 \mathrm{~ms}$. The activation wave fronts moved around the incisions, suggesting a conduction block at the isthmus. $B$, The activation map recorded by 31 bipolar electrodes during pacing at a pacing cycle length of $340 \mathrm{~ms}$. The activation time difference between electrode $A$ and electrode $B$ was 20 ms, which represents the time of travel of the stimulus wave front detouring around the incisions. The conduction velocity measured in this example represents the velocity of the pathway coming around the incision. SVC, Superior vena cava; IVC, inferior vena cava; $P V$, pulmonary vein.

however, the conduction was blocked at the isthmus and the activation detoured around the incisions. In the extremely narrow isthmi less than $5 \mathrm{~mm}$ in width, the conduction through the isthmi was blocked and the activation wave front detoured around the incisions by a roundabout pathway at all paced cycle lengths (Figure 4, A).

Conduction velocity. The conduction properties through the isthmus were characterized by the approximated curves of the conduction velocity in accordance with the pacing cycle lengths. The approximated curves of the conduction velocity were classified into 3 different types depending on the width of the isthmus (Figure 5). In the isthmi wider than $15 \mathrm{~mm}$ in width, the conduction velocity through the isthmus was constant, and slow conduction was not observed at any paced cycle length. The average conduction velocity measured $0.56 \pm 0.06 \mathrm{~m} / \mathrm{s}$ (nondecremental type). In the isthmi between 5 and $15 \mathrm{~mm}$ in width, decremental con- duction was observed (decremental type). The conduction velocity through the isthmus was within normal limits of atrial conduction velocities at long paced cycle lengths. As the paced cycle length was decreased, the conduction through the isthmus slowed. At a shorter paced cycle length, the conduction blocked at the isthmus (Figure 3,B). In the extremely narrow isthmi of less than $5 \mathrm{~mm}$ in width, the conduction velocity through the isthmus was calculated as $0.14 \pm 0.01 \mathrm{~m} / \mathrm{s}$. The conduction velocity did not change with the paced cycle lengths. Because the conduction block was confirmed by activation mapping at all of the paced cycle lengths as described above, the conduction velocity measured did not represent the velocity through the isthmus, but represented the velocity of the pathway detouring around the incisions (block type) (Figure 4, $B$ ). There were significant intergroup differences between the 3 conduction properties ( $P$ $<.01)$ by ANCOVA, with a group factor as a covariate. 


\section{Pathological Findings}

In the microscopic examination of the isthmus, 3 different types of the pathological findings were identified. These pathological findings were correlated to the conduction properties described above in all the animals. In the decremental type, fibrous tissue protruded into the surviving atrial myocardium. A small amount of surviving myocardium remained between the scars of the incisions (Figure 6, A). In the nondecremental type, a larger amount of atrial myocardium remained at the isthmus compared with that of the decremental type. In the block type, all of the atrial myocardium at the isthmus was replaced by fibrous tissue, and no surviving myocardium was observed between the incisions (Figure 6, $B$ ).

In all 16 animals, in which isthmi of various widths were made, there was a significant positive correlation between the width of the isthmus determined preoperatively and the area of the surviving myocardium at the isthmus determined microscopically after the experiment (Figure 7).

\section{Discussion}

This animal study demonstrated that there were 3 types of conduction properties through the isthmus between the tandem incisions made on the lateral right atrium. The width and volume of the surviving atrial myocardium in the isthmus determined the conduction property. In the isthmi between 5 and $15 \mathrm{~mm}$ in width, the amount of surviving atrial myocardium was decreased to a critical level, which resulted in slow conduction through the isthmus during rapid stimulation. This characteristic property of the conduction through the isthmus may facilitate the sustenance of the IART.

The present study suggested that the slow conduction in the critical isthmus between the atrial scars plays an important role in the development of the IART. Nakagawa and colleagues demonstrated reentrant activation conducting through the narrow isthmus between surgical scars by electroanatomical mapping in 16 patients who developed IART after surgical repair of congenital heart disease. ${ }^{12}$ The pathological findings in our study have suggested that the critical isthmus is formed by a small amount of surviving atrial myocardium between the scars. Because of the scarce viable myocardium, the activation barely passes through the isthmus, resulting in the slow conduction and setting up the reentrant circuit of the IART. Scarring is an additional factor in the formation of slow conduction in the isthmus. The microscopic findings in the present study have shown that fibrous tissue protrudes into the surviving atrial myocardium at the isthmus, further narrows the isthmus, and decreases the conductivity of the isthmus. In the lateral right atrium after surgery for congenital heart disease, where multiple incisions and stitches are made, scar formation is induced in the atrial myocardium by the same mechanism.

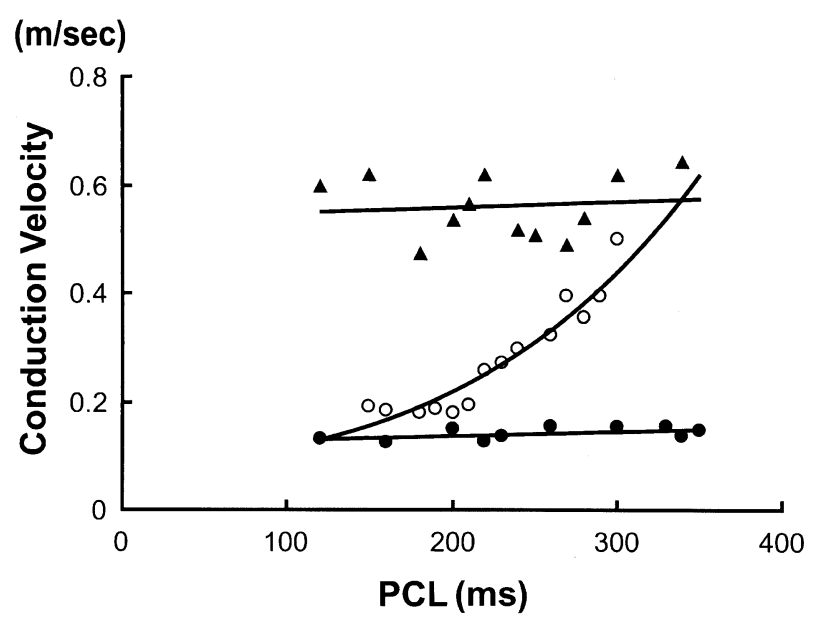

Figure 5. The relationship between the conduction velocity and paced cycle length. Three different types of plots and approximated curves are confirmed. The pattern of the plots and curves signifies the conduction properties thorough the isthmus. The closed triangles represent the conduction property through an isthmus $15 \mathbf{~ m m}$ in width. The conduction velocity is constant at any paced cycle length (nondecremental type). The open circles represent the conduction property through an isthmus $12 \mathrm{~mm}$ in width. The pattern of conduction shows decremental conduction (decremental type). The closed circles represent the block type conduction (isthmus $3 \mathrm{~mm}$ in width). There were significant intergroup differences between the 3 conduction properties $(P<$ .01). See the text for details. PCL, Paced cycle length.

Furthermore, in an isthmus with a certain range of width, the amount of surviving myocardium is decreased to a critical level, setting up for slow conduction.

Wave-front curvature and propagation around the sharp edges of incisions have also been shown to play another important role in maintaining the IART. ${ }^{14,15}$ The activation pivots around the edge of an anatomical barrier during reentrant activation. The conduction slows at the pivot point fulcrum, whereas conduction is fast at sites near to or distant from the pivoting fulcrum. ${ }^{16}$ The slow conduction at the pivot point allows for ample time for the refractory period of the atrial myocardium to recover. Therefore, it is considered that the curvature of the wave front at the pivot also gets involved in the mechanism of the stable sustenance of IART.

The incidence of IART has been reported to be higher, ranging from $20 \%$ to $37.5 \%$, for follow-up periods of up to 15 years after the Fontan procedure than that after surgery for atrial septal defect or tetralogy of Fallot. ${ }^{7,17}$ The factors predisposing the post-Fontan patients to IART include not only the atrial scarring caused by the multiple atriotomies but also the elevated atrial wall stress or atrial hypertrophy resulting from the increased atrial pressure and volume. The patients with a large atrial size and volume have been shown 

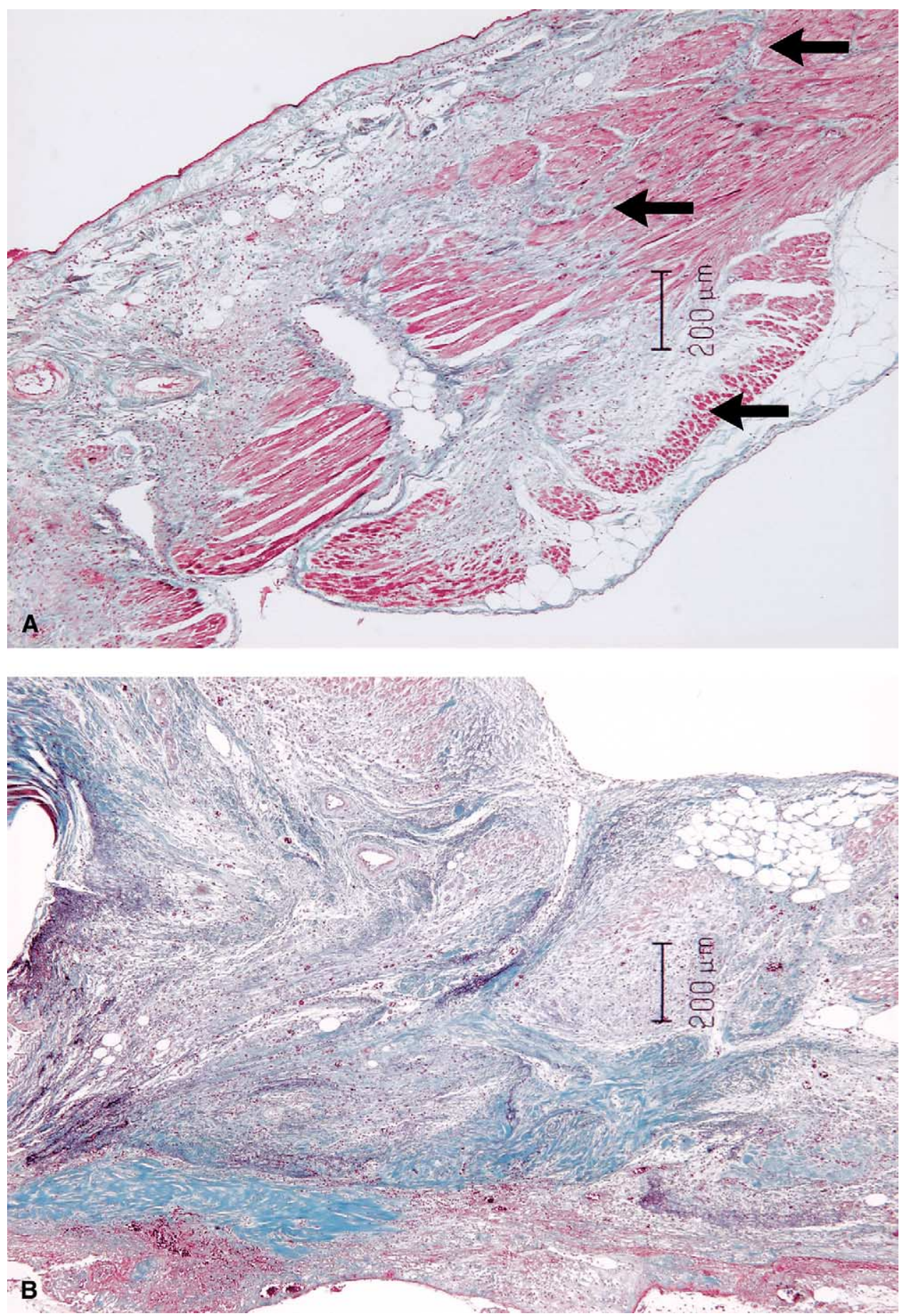

Figure 6. Photomicrographs of an isthmus between incisions. A, The arrows represent elastic fibers protruding into the atrial surviving myocardium at an isthmus of $12 \mathrm{~mm}$ in width. Note that a small amount of surviving myocardium remains between the scars of the incisions. Elastica-Masson-Goldner stain: magnification $\times 4.0$; bar $=200 \mu \mathrm{m}$. $B$, The entire atrial myocardium at the isthmus is replaced by fibrous tissue for an isthmus of 3 mm in width. Elastica-Masson-Goldner stain: magnification $\times 4.0$; bar $=200 \mu \mathrm{m}$. 
to have a higher incidence of IART than those with a normal range of atrial size after the Fontan procedure. ${ }^{6}$ Non-transmural suture lines like those in the lateral tunnel procedure also cause slow conduction through the suture lines. Rodefeld and colleagues described that multiple reentrant circuits crossed over non-transmural suture lines in an experimental study. ${ }^{11}$ The post-Fontan patients have multiple isthmi in their right atrium, while the patients after surgery for atrial septal defect or tetralogy of Fallot usually have only 1 isolated isthmus, which can cause slow conduction.

Although the present study was not conducted to examine the inducibility of IART, it was suggested that IART could be prevented by leaving a sufficient amount of surviving myocardium in the isthmus or by connecting the incisions to avoid conduction through the isthmus. In a small infant, however, it should be impossible to keep a distance of more than $15 \mathrm{~mm}$ between the incision and the cannulation site on the small right atrium. One option should be employing a bicaval cannulation technique, inserting cannulas directly into the superior vena cava and inferior vena cave, to avoid multiple incisions of the right atrium. If multiple incisions are placed on the right atrium during the initial surgery, the incisions should be connected to each other by an ablative procedure to eliminate leaving any isthmi. Haas and colleagues described that no patients had cardiac arrhythmias after a median follow-up of 64 months after an extracardiac conduit Fontan procedure by observation using Holter monitoring. ${ }^{18}$ The extracardiac conduit Fontan procedure has fewer atrial incisions than the traditional Fontan procedure. Therefore, a modification of the Fontan procedure, which does not have a critical isthmus, may lower the incidence of IART.

In addition to the width of the isthmus, growth of pediatric patients should be also considered as a determinant for the conduction property through the isthmus. It has been described that the incidence of IART is higher as time has passed after surgery than early after the surgery. ${ }^{6}$ The incidence of late IART after surgery has been shown to be $6 \%$ by 1 year, $12 \%$ by 3 years, and $17 \%$ by 5 years in 499 patients after the Fontan procedure. ${ }^{19}$ The width of the isthmus and the amount of the survival atrial myocardium at the isthmus may change to a critical level sufficient to allow IART to develop as pediatric patients grow up.

The direction of the atrial myocardium in accordance with the incisions should also be considered in the perpetuation of IART. Spach and colleagues have shown that transverse propagation is slower than longitudinal propagation in the direction of atrial myocardial fibers. ${ }^{20}$ If the incisions are drawn in a direction parallel to atrial myocardium on the right atrium, the conduction velocity through the isthmus would be extremely slow. In the present study, we placed the incision lines on the lateral right atrium between the right atrial appendage and the sinus node artery

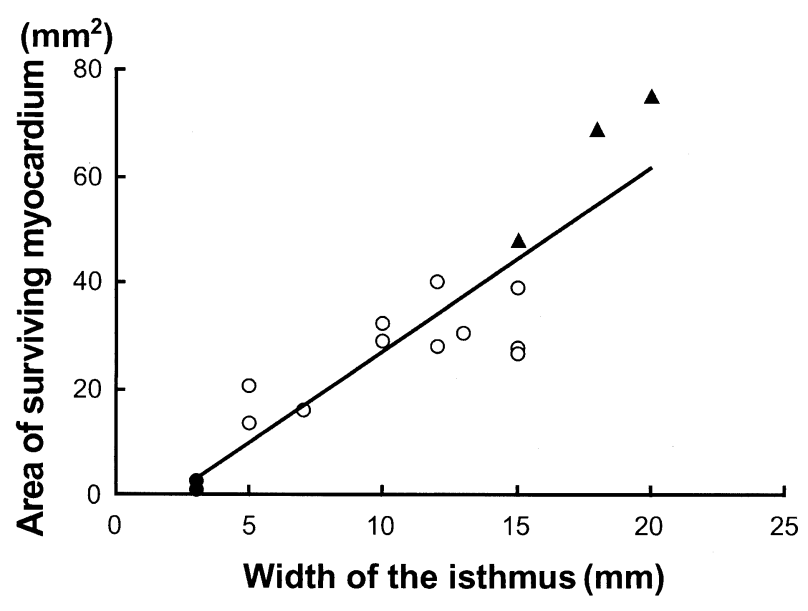

Figure 7. Correlation between the width of the isthmus determined during the initial surgery and the area of the surviving myocardium at the isthmus determined microscopically 4 weeks after the initial surgery. $y$ axis, surviving myocardium area in $\mathrm{mm}^{2} ; x$ axis, width of the isthmus in $\mathrm{mm} ; y=0.23 x+3.99 ; \mathrm{r}$ (correlation coefficient) $=0.89, P<.001$. There was a linear correlation between the width of the isthmus and the area of the surviving myocardium. Closed circles, open circles, and closed triangles represent the block type, decremental type, and nondecremental type, respectively.

in all canines and did not examine the relationship between the direction of incisions and myocardial fiber direction. This relationship should be examined in detail, because it would affect the incidence of IART and would be helpful in designing an atrail incision that would avoid the occurrence of IART in surgery for congenital heart disease.

We acknowledge the excellent technical assistance of Motoaki Uchiyama. We also thank John Martin for preparation of the manuscript.

\section{References}

1. Girod DA, Fontan F, Deville C, Ottenkamp J, Choussat A. Long-term results after the Fontan operation for tricuspid atresia. Circulation. 1987;75:605-10.

2. Gelatt M, Hamilton RM, McGrindle BW, et al. Risk factors for atrial tachyarrhythmias after the Fontan operation. J Am Coll Cardiol. 1994; 24:1735-41.

3. Bink-Boelkens MThE, Velvis H, Homan van der Heide JJ, et al. Dysrhythmias after atrial surgery in children. Am Heart J. 1983;106: 125-30.

4. Murphy JG, Gersh BJ, McGoon MD, et al. Long-term outcome after surgical repair of isolated atrial septal defect: follow-up at 27 to 32 years. N Engl J Med. 1990;323:1645-50.

5. Peters NS, Sommerville J. Arrhythmias after the Fontan procedure. $\mathrm{Br}$ Heart J. 1992;68:199-204.

6. Ghai A, Harris L, Harrison DA, et al. Outcomes of late atrial tachyarrhythmias in adults after the Fontan operation. J Am Coll Cardiol. 2001;37:585-92.

7. Balaji S, Johnson TB, Sade RM, Case CL, Gillette PC. Management of atrial flutter after the Fontan Procedure. J Am Coll Cadiol. 1994;23: 1209-15. 
8. Kalman J, Van Hare GF, Olgin J, et al. Ablation of "incisional" reentrant atrial tachycardia complicating surgery for congenital heart disease: use of entrainment to define a critical isthmus of conduction. Circulation. 1996;93:502-12.

9. Lesh MD, Kalman JM, Saxon LA, Dorostkar PC. Electrophysiology of "incisional" reentrant atrial tachycardia complicating surgery for congenital heart disease. PACE. 1997;20:2107-11.

10. Gandhi SK, Bromberg BI, Rodefeld MD, et al. Lateral tunnel suture line variation reduces atrial flutter after the modified Fontan operation. Ann Thorac Surg. 1996;61:1299-309.

11. Rodefeld MD, Bromberg BI, Schuessler RB, et al. Atrial flutter after lateral tunnel construction in the modified Fontan operation: a canine model. J Thorac Cardiovasc Surg. 1996;111:514-26.

12. Nakagawa H, Shah N, Matsudaira K, et al. Characterization of reentrant circuit in macroreentrant right atrial tachycardia after surgical repair of congenital heart disease: isolated channels between scars allow "Focal" ablation. Circulation. 2001;103:699-709.

13. Lee R, Nitta T, Schuessler RB, et al. The closed heart MAZE: a nonbypass surgical technique. Ann Thorac Surg. 1999;67:1696-702.

14. Cabo C, Pertsov AM, Baxter WT, et al. Wave-front curvature as a cause of slow conduction and block in isolated cardiac muscle. Circ Res. 1994;75:1014-28.

15. Fast VG, Kleber AG. Role of wavefront curvature in propagation of cardiac impulse. Cardiovasc Res. 1997;33:258-71.

16. Girouard SD, Pastore JM, Laurita KR, et al. Optical mapping in a New Guinea pig model of ventricular tachycardia reveals mechanisms for multiple wavelengths in a single reentrant circuit. Circulation. 1996; 93:603-13.

17. Porter CJ, Garson A. Incidence and management of dysrhythmias after Fontan procedure. Herz. 1993;18:318-27.

18. Haas GS, Hess H, Black M, Onnasch J, Mohr FW, van Son JAM. Extracardiac conduit Fontan procedure: early and intermediate results. Eur J Cardiothorac Surg. 2000;17:648-54.

19. Durongpistikul K, Porter CJ, Cetta F, et al. Predictors of early- and late-onset supraventricular tachyarrhythmias after Fontan operation. Circulation. 1998;98:1099-107.

20. Spach MS, Miller WT, Geselowitz DB, Barr RC, Kootsey JM, Johnson EA. The discontinuous nature of propagation in normal canine cardiac muscle. Circ Res. 1981;48:39-54. 